\title{
EVALUACIÓN DE COMPETENCIA DEL NIVEL BACHILLER: "ASEGURAMIENTO DE LA CALIDAD EN LA FORMACIÓN DE ESTUDIANTES DE ENFERMERÍA"
}

\author{
COMPETENCE ASSESSMENT AT BACHELOR LEVEL: QUALITY \\ ASSURANCE IN NURSING STUDING STUDENTS TRAINING
}

\author{
Claudia Andrea Muñoz Jofré* \\ Cecilia Latrach Ammar ${ }^{* *}$ \\ IRENE GONZÁLEZ VACAREZZA** \\ Mabel Araya NovoA ${ }^{* * *}$
}

\begin{abstract}
RESUMEN
El propósito de este trabajo es contribuir al aseguramiento de la calidad de la formación profesional de enfermería a través de la evaluación de competencias. Objetivo: Construir un método de evaluación de competencias del Nivel de Bachiller de la Escuela de Enfermería como plan piloto y modelo que permita garantizar la calidad de la formación del futuro profesional de enfermería. Métodos: Se estructuró en cuatro etapas: Diseño sistema de evaluación, ejecución del proceso evaluativo, análisis de resultados, control y evaluación. Universo de 71 estudiantes de segundo año $(n=67) 2 \%$ ES. Se evaluó desempeño de una competencia asistencial en contextos reales hospitalarios aplicando rúbrica validada con 14 actividades de enfermería. Se categorizó a los estudiantes según desempeño de excelencia, efectivo y que requiere mejoramiento. Resultados: El $41 \%$ de los estudiantes tuvo un desempeño efectivo, $31 \%$ desempeño excepcional y un $28 \%$ requiere mejoramiento. Conclusiones: $\mathrm{El}$ sistema de evaluación fue validado, la rúbrica facilitó la categorización del nivel de desempeño; un tercio de los estudiantes debe realizar medidas remediales para mejorar desempeño.
\end{abstract}

Palabras clave: Competencias, evaluación, calidad.

\begin{abstract}
The purpose of this study is to contribute to the quality assurance of professional nurses training through the competency assessment. Objective: To design a competency assessment method level BS from the School of Nursing as a pilot and model to ensure the quality of education of future nurses. Methods: The study was structured in four stages: design evaluation system, performance evaluation process, analysis of the results, monitoring and evaluation. Universe of 71 students in second year $(n=67) 2 \%$ ES. We evaluated skills performance of a health care competency in real hospital context applying a validated Heading in 14 nursing activities. Students were categorized according to performance of excellence, effective and require improvement. Results: $41 \%$ of students had an effective performance, $31 \%$ exceptional performance and a $28 \%$ improvement required. Conclusions: The evaluation system was validated, heading facilitated the categorization of the level of performance, one third of the students must undertake remedial measures to improve performance.
\end{abstract}

Key words: Competencies, assessment, quality.

Fecha recepción: 29/10/08 Fecha aceptación: 16/11/09

\footnotetext{
* Enfermera, académica de Escuela de Enfermería Universidad Mayor. Santiago, Chile. E-mail: claudia.munozj@umayor.cl

${ }^{*}$ Enfermera, académica de Escuela de Enfermería Universidad Mayor. Santiago, Chile. E-mail: cecilia.latrach@umayor.cl

${ }^{* * *}$ Enfermera, académica de Escuela de Enfermería Universidad Mayor. Santiago, Chile. E-mail: irene.gonzalez@umayor.cl

${ }^{* * * *}$ Enfermera, académica de Escuela de Enfermería Universidad Mayor. Santiago, Chile. E-mail: mabel.araya@umayor.cl
} 


\section{INTRODUCCIÓN}

La sociedad está viviendo en estas últimas décadas una serie de transformaciones producto de la globalización, el desarrollo tecnológico, cambios en los paradigmas educativos y una explosión desbordante de descubrimientos y progresos científicos (1). Como lo comenta Arata (2), esto indudablemente ha significado y significará una evolución cultural de una magnitud inmensurable. En este contexto, las universidades han experimentado cambios y algunos de los más significativos, según señala Mérida (3), es la existencia de una nueva cultura de la calidad; entre éstos un modelo de enseñanza centrado en los procesos de aprendizaje del estudiante y demandas del mundo laboral de acuerdo a las necesidades reales de la población. En la actualidad ya no se exige sólo un gran caudal de conocimientos sino también competencias especializadas y habilidades personales más genéricas que permiten la formación continua.

Según Cardenal (4) señala que los estudiantes expresan fe en el sistema de enseñanza como dispositivo de colocación social, por lo que es un imperativo ético de la formación universitaria el asegurar la calidad de la formación para su futuro desempeño laboral.

Hawes y Corvalán (5) señalaron que "La formación profesional, en el marco del contexto nacional, está en proceso de renovación, diferentes instituciones han iniciado cambios en mayor o menor profundidad desde hace algunos años". Este es el escenario actual en el que se están desenvolviendo algunas universidades chilenas y que tienen hoy como desafío enfrentar con éxito la formación en ámbitos cambiantes y la responsabilidad de formar profesionales que den respuesta de forma competente a las necesidades del país, es necesario entonces, revisar el sentido y las prácticas educativas que se desarrollan en cada ámbito universitario que se orienten al aseguramiento de la calidad de la formación, aspecto que es parte de las metas del bicentenario en educación superior en Chile (6).

Se destaca el proceso de aseguramiento de la calidad de las instituciones de Educación Superior, donde la acreditación institucional a cargo de la Comisión Nacional de Acreditación de Pregrado adquirió relevancia ya que ésta se centra en la existencia y operación eficaz de políticas y mecanismos para verificar el cumplimiento de los propósitos institucionales, en un marco general de criterios de calidad (7).

Para algunas entidades superiores como la Universidad Austral de Chile, Pontificia Universidad Católica de Chile, Universidad de Santiago, entre otras, el actual modelo educativo chileno hace mención al enfoque curricular basado en competencias, donde la eficiencia de los procesos formativos garantizan la formación integral y de excelencia considerando salidas intermedias a nivel bachillerato y licenciatura junto con enlazar el pregrado-posgrado (8).

Se define competencia como "Capacidad de lograr cierto objetivo en un contexto dado, lo cual implica poseer y desarrollar conocimientos, destrezas, habilidades y actitudes que le permiten a una persona realizar actividades inherentes a su profesión, adaptarse a nuevas situaciones y transferir sus conocimientos, habilidades y actitudes en algunas ocasiones o situaciones profesionales no tan inherentes a su profesión" (9). Sin embargo, cuando se evalúan competencias los criterios responden al qué evaluar y no al qué enseñar, estos criterios evalúan aprendizajes básicos sin que los alumnos difícilmente podrían seguir de forma satisfactoria su proceso de aprendizaje; al analizar el curriculum básico se hace hincapié en el logro y evaluación de competencias del perfil del egresado, sin embargo no se señala como hacerlo (10). 
El bachillerato es definido como el conjunto de cursos y actividades académicas con una secuencia curricular coherente, con objetivos tales como entregar educación universitaria básica que permita integrar estudios superiores o enfrentar el campo laboral, como también poner a prueba sus reales competencias para continuar su formación profesional (11).

En este marco la Universidad Mayor, en el año 2004, implementó un currículum basado en competencias; en su malla curricular existen cuatro bloques: Inicial, Disciplinario, Profesional y Especializado. Al final del bloque inicial se habilita al estudiante para recibir el Grado de Bachiller y la Certificación de Competencias correspondiente y así al concluir cada bloque (12).

La Escuela de Enfermería de la Universidad Mayor para el levantamiento de su perfil de egreso y las competencias del nivel bachillerato licenciatura y profesionales, consideró los cambios en la realidad de salud del país, el nuevo perfil epidemiológico y el sistema de salud chileno reformado (13).

Según Torres (14) existe un vínculo muy estrecho entre perfil, funciones y competencias, esto fue evidenciado durante el proceso de identificación de competencias que realizó la carrera a través del modelo de análisis funcional, método que desagrega el propósito principal de la carrera en funciones y subfunciones hasta llegar a identificar unidades de competencias $(14,15)$.

Con este currículum renovado la tarea docente se orienta a desarrollar competencias genéricas y específicas en los estudiantes a través de metodologías innovadoras que enriquezcan su aprendizaje en un contexto particular. Es necesario distinguir las capacidades, conocimientos, habilidades, destrezas y actitudes que son parte del perfil de competencias necesarias para el ejercicio de la profesión de las futuras egresadas y egresados de la carrera y que orientan el foco de la formación profesional, como así mismo, los escenarios pedagógicos para la construcción de competencias, su posterior evaluación y certificación garantizando con esto la calidad de la educación que se entrega. Como se plantea (16) las competencias se capitalizan, se suman y resulta el perfil profesional. Este perfil por competencias debe insertarse en una "realidad dinámica y móvil, que está en permanente cambio y ajuste en relación con el entorno y sus variaciones" (5).

Un estudio realizado en Chile y México observó diferencias en las competencias de los distintos niveles de preparación académica superior con relación a los ítemes de conocimiento y tendencias de la investigación (17).

Otro estudio realizado en Cuba analiza los resultados de los exámenes de competencia en el país durante los años 1997 y 1999 tanto en enfermeros y técnicos como en licenciados, siendo los resultados satisfactorios de un 90\% en el año 1997; la de mayor dificultad encontrada fue en las temáticas de esterilización, desinfección y proceso de atención de enfermería de estos 3 años estudiados (18).

Otro estudio descriptivo realizado en nueve facultades de escuelas de enfermería de países latinoamericanos realizado a través de un instrumento estructurado enviado por vía electrónica demuestra un perfil de licenciado orientado a un profesional generalista capacitado para la asistencia integral del individuo familia y comunidad, con conocimientos, gestión, investigación y educación (19).

A su vez el mundo laboral ha implementado un modelo que orienta al desarrollo de competencias y se basa en tres subsistemas de selección, desarrollo y evaluación de desempeño (20). En este nuevo escenario los profesionales de enfermería a futuro se verán sometidos a evaluación de competencias en concordancia con lo que explicita esta profesión en Chile como una disciplina 
a través del reconocimiento legal de la profesión de enfermera en el artículo 113 del Código Sanitario en 1997 (21) y la inclusión formal del ejercicio profesional al marco jurídico de la Reforma de salud en el año 2005 a través de la Gestión del cuidado de enfermería (22).

En la actualidad empresas del rubro salud realizan evaluación de desempeño de sus empleados midiendo cumplimiento de competencias laborales. Por lo que esta instancia sirve de experiencia a los alumnos ya que los prepara para enfrentarse a este tipo de evaluaciones (23).

Este trabajo pretende ser un aporte a la disciplina de enfermería, ya que al contar con procesos de evaluación de competencias en el nivel de bachiller se fortalecen los mecanismos de aseguramiento de la formación de profesionales.

\section{OBJETIVO GENERAL}

Evaluar competencias del nivel de bachiller de la Escuela de Enfermería de la Universidad Mayor que permita garantizar un aseguramiento continuo de la calidad de la formación del futuro profesional de enfermería.

El proceso de evaluación de competencias del nivel de Bachiller se estructuró en 4 etapas:

Etapa 1. Diseño del sistema de evaluación de competencias de nivel Bachillerato en Enfermería donde se realizó la selección de la competencia asistencial para el nivel $\mathrm{Ba}$ chiller desde el mapa funcional, identificando los aprendizajes esperados que dan respuesta a la competencia, fijación de criterios de evaluación y de estándares de desempeño, selección y confección de instrumento de evaluación; todo el proceso fue asesorado por equipo experto en competencias Dirección de Desarrollo Académico (DDA) - Sistema de Certificación de Competencias (SCC).

\section{Etapa 2. Ejecución de proceso evaluativo.}

El Equipo evaluador estuvo constituido por enfermeras docentes encargadas de los alumnos en campos clínicos, a quienes se realizó un proceso de inducción. El instrumento se elaboró en base a rúbricas con 14 actividades a evaluar según criterios y con 3 categorías de desempeño: excepcional, efectivo y que requiere mejoramiento. El Instrumento fue validado por juicio de expertos y cuenta con un nivel de confiabilidad de 0,88 (Alpha de Cronbach). Se evaluó a los estudiantes en diferentes centros hospitalarios con pacientes reales aplicando una rúbrica de competencias durante el año 2007. El universo estuvo constituido por 71 estudiantes de segundo año de la carrera de enfermería, y la muestra fue de 67 estudiantes seleccionada con un error estándar de $2 \%$ a un nivel de confianza del $95 \%$.

Etapa 3. Proceso de análisis de resultados evaluativos. Se realizó revisión y validación de evaluaciones efectuadas por los docentes.

Etapa 4. Proceso de control y evaluación. Se efectuó evaluación y validación de Plan Piloto por expertos en competencias de la Dirección General Académica (DGA) de la universidad y retroalimentación de resultados. Se planificaron medidas remediales para el año siguiente. 


\section{RESULTADOS}

Gráfico 1. "Nivel de desempeño global de la competencia asistencial por los estudiantes de enfermería” ( $n=67)$.

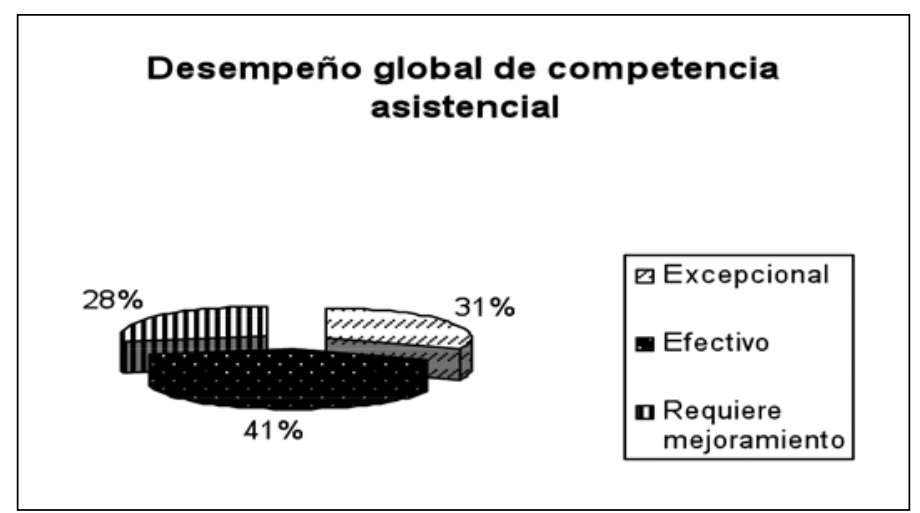

Se observó que el $41 \%$ de los estudiantes logró desempeño efectivo, 31\% desempeño excepcional y un $28 \%$ logró un desempeño que requiere mejoramiento. Esto mues- tra que la mayoría de ellos es competente en el nivel de salida de bachiller y un tercio de ellos requiere mejoramiento.

Gráfico 2. "Nivel de desempeño por actividades de los estudiantes de enfermería”.

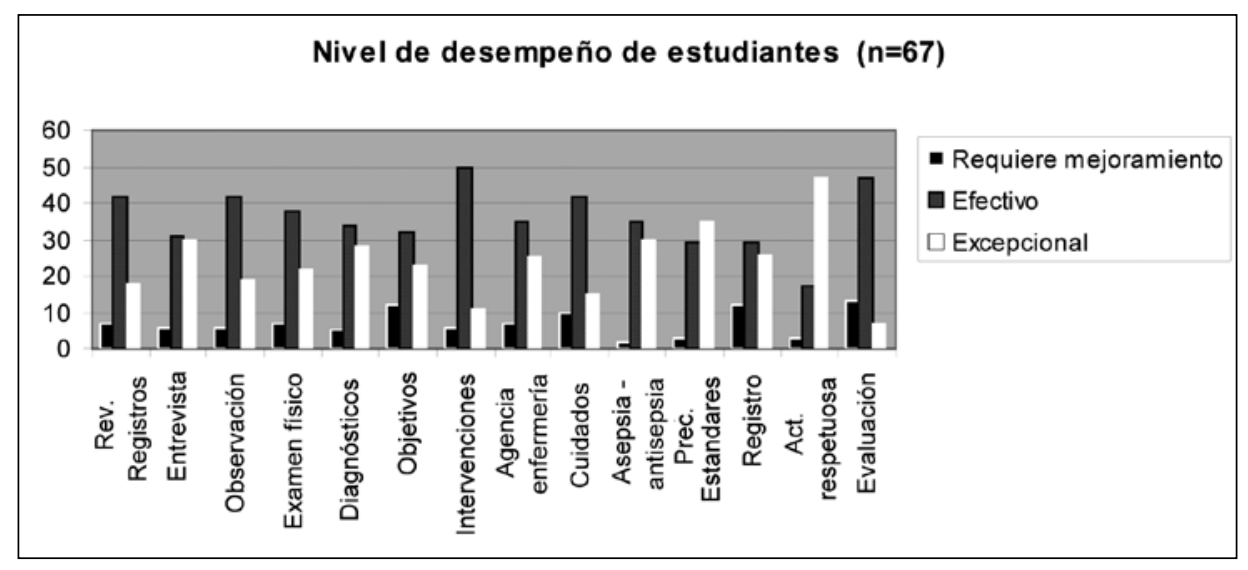

Las actividades donde los estudiantes lograron el desempeño con porcentajes del 95\% considerando el desempeño efectivo y el de excelencia fueron "utilizar principios de asepsia y antisepsia” y "aplicación de precauciones estándar”.

En todas las actividades que se realizan en la etapa de valoración: "revisión de registros”, "realizar entrevista al usuario", "observación" y el "examen físico" lo lograron sobre el 90\%, igualmente en la "Identificación de problemas o diagnósticos de enfermería".

En las otras actividades de la etapa de planificación, ejecución y evaluación, están so- 
bre el $80 \%$ de logro.

Importa destacar que en las actividades, "Elaborar objetivos", "Realizar registros" y "Realizar evaluación" hay un porcentaje mayor de alumnos que requiere mejoramiento.

\section{DISCUSIÓN Y CONCLUSIONES}

El estudio permitió medir el desempeño de los aprendizaje esperados que el estudiante tiene en el nivel de bachiller y orientar la aplicación de medidas remediales con aquellos que no lograron la competencia en este nivel, lo que asegura la calidad de la formación.

Los estudios encontrados $(17,18)$ hacen referencia a la identificación de competencias profesionales según nivel, sin abocarse a la evaluación de competencia específicas, motivo por el cual no es posible su contrastación; asimismo el estudio descriptivo realizado en las nueves escuelas de Enfermería (19) orienta hacia las competencias del perfil del licenciado, el cual no mide competencias específicas y tampoco hace referencia a la evaluación en el nivel de bachiller, por ende difieren de este estudio tanto en el objetivo como en la metodología de recolección de datos.

El $72 \%$ de los estudiantes de Bachiller consiguió en forma efectiva el desempeño de la competencia, un tercio requirió mejoramiento para su logro y el desempeño excepcional fue obtenido por un porcentaje menor.

El usar la rúbrica en el instrumento de evaluación de competencias facilitó una evaluación precisa y objetiva sin lugar a interpretaciones o juicios por parte del evaluador y facilitó la categorización del nivel de desempeño de los estudiantes. Este sistema de evaluación permitió medir el logro de la competencia seleccionada, considerando que ésta implica por definición conocimientos, habilidades, destrezas y actitudes.

Esta experiencia permite replicar el mo- delo de evaluación de competencias en los otros niveles de salida, licenciado y egresado, con la finalidad de asegurar los aprendizajes, con esto la calidad en la formación y por lo tanto el aporte al mercado laboral e instituciones de salud.

\section{IMPLICANCIAS Y PROYECCIONES}

El evaluar competencias de formación del nivel bachiller favorece una mejora continua del proceso de formación de los futuros profesionales enfermeras/os, esto permite implementar intervenciones oportunas en beneficio de la calidad educacional superior junto con cumplir con los estándares incorporados a los aprendizajes, que se espera adquieran los estudiantes en este período de educación profesional.

Las grandes transformaciones sociales y económicas de un mundo globalizado exigen de profesionales con competencias laborales que respondan a la necesidades actuales, para esto es importante que los estudiantes estén vinculados a procesos de evaluación, que certifiquen habilidades individuales y competencias actualizadas necesarias para desempeñarse efectivamente en la sociedad, mecanismos cada vez más utilizados en los países desarrollados que garantizan la seguridad y la calidad de la atención en salud.

\section{REFERENCIAS}

1. Delors J. La educación encierra un tesoro. Informe a la UNESCO de la Comisión Internacional sobrela educación para el siglo XXI. Ediciones Unesco [Seriada en línea] 1997. Hallado en: http://unesdoc.unesco. org/images/0010/001095/109590So.pdf [Acceso en junio 2008].

2. Arata A. Excelencia académica y gestión universitaria, una combinación posible y 
necesaria. Universidad Técnica Federico Santa María. $1^{\text {a }}$ ed. Valparaíso, Universidad Técnica Federico Santa María. 2001.

3. Mérida R. La convergencia europea y la formación universitaria en competencias para la docencia en Educación Infantil. Rev. Educ [en línea] 2006; (341):663-86. Hallado en: http://www.revistaeducacion.mec.es/re341/re341_27. pdf [Acceso en junio 2008].

4. Cardenal, M. La universidad como dispositivo de colocación social. Movilidad y reproducción en la era de la precariedad laboral. Rev. Educ. [Seriada en línea] 2006; (341):281-299. Hallado en: http:// www.revistaeducacion.mec.es/re341/ re341_13.pdf [Acceso en junio 2008].

5. Hawes, Corvalán O. Construcción de un perfil profesional. [Documento de trabajo 1/2004]. 2005. Universidad de Talca. Instituto de investigación y desarrollo institucional Proyecto MECESUP TALO 101 [en línea] Hallado en: http://www. mecesup.cl/mecesup1/difusion/destacado/2004-1\%20Construccion $\% 20 \mathrm{de} \% 20$ un $\% 20$ Perfil\%20Profesional.pdf [Acceso en junio 2008].

6. González J, Wagenaar R, Beneitone P. Tuning América Latina: Un proyecto de las universidades. Rev Iberoam Educ [en línea] 2005: (35). Hallado en: http://www. rieoei.org/rie35a08.html [Acceso en junio 2008].

7. Lemaitre M. Aseguramiento de la calidad en Chile. Impacto y proyecciones. Comisión Nacional de Acreditación de Pregrado [Ponencia] Congreso internacional retos y expectativas de la universidad. México. 2006. [en línea] Hallado en: http://www.congresoretosyexpectativas.udg.mx/Congreso\%206/Conferencias\%20Magistrales/Mesa3/marialemaitre.pdf [Acceso en junio 2008].

8. Cubillos V. Discurso 55 aniversario Universidad Austral de Chile 2006. Hallado en: http://www.uach.cl/rrpp/online/ anexos/2009/09/discursorectorceremo- nialcentral.pdf [Acceso en junio 2008].

9. Universidad Mayor. Dirección General Académica. Competencias en la U. Mayor. [8 Diapositivas]. Universidad Mayor Sede Huechuraba. 2005. Santiago, Chile.

10. Ramírez Y. El perfil de competencias y la evaluación cualitativa del aprendizaje en la I y II etapas de educación básica. EDUCERE [en línea] 2004; (25): 159-166. Hallado en: http://redalyc.uaemex.mx/redalyc/src/inicio/ArtPdfRed. jsp?iCve $=35602504$ [Acceso en junio 2008].

11. López J. El bachillerato: una innovación curricular mayor en la Pontificia Universidad Católica de Chile [en línea]. Hallado en: http://www.cse.cl/public/ secciones/seccionpublicaciones/doc/43/ cse_articulo309.pdf [Acceso en junio 2008].

12. Universidad Mayor. La Visión emprendedora de la Mayor ahora cuenta con el CMY Currículum Mayor. Presentación Currículum Mayor. 2004. Santiago, Chile.

13. Ministerio de Salud (MINSAL). Encuesta Nacional de Salud. Gobierno de Chile. 2004. Hallado en: http://epi.minsal.cl/ epi/html/invest/ENS/ENS_mayo2004. pdf [Acceso en junio 2008].

14. Torres E, Urbina L. Perfiles profesionales, funciones y competencias del personal de Enfermería en Cuba [en línea]. Educ Med Super. 2006;20(1): (Scielo).

15. Irigoin M, Vargas F. (2002). Competencia Laboral: manual de conceptos, métodos y aplicaciones en el sector salud. Módulo 1. Competencias, fases y aplicación. Unidad 3. Identificación y normalización de competencias [en línea]. Montevideo: Cinterfor-OPS. Hallado en: http://www. ilo.org/public/spanish/region/ampro/ cinterfor/publ/man_ops/pdf/mod1_3. pdf [Acceso junio 2008].

16. Corominas E, Tesouro M, Capell D. Percepciones del profesorado ante la incorporación de las competencias genéricas 
en la formación universitaria. Rev Educ. [en línea]. 2006 (341): 301-336. Hallado en: http://www.revistaeducacion.mec. es/re341/re341_14 [Acceso noviembre 2008].

17. Harrison L, Ray A, Cianelli R, Rivera M, Urrutia M. Competencias en investigación para diferentes niveles de formación de enfermeras: una perspectiva latinoamericana. Cienc y Enferm. 2005; 11(1): 59-71.

18. Urbina O, Barazal A. Experiencia de la evaluación de la competencia profesional en enfermería en el período 1997-1999. Rev Cubana de Educ Med Super. 2002; 16(2): 120-7.

19. Nájera R, Jara $P, B$ Ben V. Innovaciones en la formación del licenciado en enfermería en Latinoamérica, al inicio del siglo XXI. Investigación y educación en enfermería. 2002; 20(2):48-56.
20. Alles M. Desarrollo del talento humano basado en competencias. Editorial Granica. Buenos Aires. 2006.

21. Código sanitario DFL N725/67. Publicado en Diario Oficial 31.01.68. Hallado en: http://www.minsal.cl/juridico/ DFL_725_DE_1969.doc [Acceso en diciembre 2008].

22. Colegio de Enfermeras. La importancia de un reconocimiento legal [en línea]. Hallado en: http://www.colegiodeenfermeras.cl/datos/ftp/reconocimiento_legal_enfermeria.pdf [Acceso diciembre 2008].

23. Chilecalifica. La formación basada en competencias en América Latina y el Caribe. Desarrollo reciente. Algunas Experiencias. Enero 2004. Hallado en: http:// www.chilecalifica.cl/califica/Noticias/ NoticiaDesplegada/metropolitana/noticia020 [Acceso diciembre 2008]. 